\title{
Pilot Study of Wobble Seat on Trunk Muscles Activity and Kinematics
}

\author{
Ieva ALEKNAITE-DAMBRAUSKIENE*, Aurelijus DOMEIKA**, Vaidotas GUDŽIŪNAS***, \\ Vidmantas ZAVECKAS**** \\ *Kaunas University of Technology, Studentu 56, 51424 Kaunas, Lithuania, E-mail: ieva.aleknaite-dambrauskiene@ktu.lt \\ **Kaunas University of Technology, Studentu 56, 51424 Kaunas, Lithuania, E-mail: aurelijus.domeika@ktu.lt \\ ***Kaunas University of Technology, Studentu 56, 51424 Kaunas, Lithuania, E-mail: v.gudziunas@gmail.com \\ ****Lithuanian University of Health Sciences, Tilžès 18, 47181 Kaunas, Lithuania, \\ E-mail:vidmantas.zaveckas@gmail.com
}

crossref http://dx.doi.org/10.5755/j02.mech.26612

\section{Introduction}

Insufficient lumbar stability is known as a potential mechanism which is associated with disability and lower back pain [1]. The motor control challenge for the individuals is to use their lumbar spine to minimize the seat displacement, when sitting on unstable surface, with smaller movements representing better performance [1]. This requires a good connection between feedforward and feedback motor control mechanisms. Feedforward control can change lumbar stiffness because of trunk muscle co-contraction. However, too much trunk muscle co-contraction can reduce trunk postural control during unstable sitting. Other studies also show, that individuals with non - specific lower back pain have shown poorer postural control during sitting [2]. During standing and sitting, impaired proprioception has been suggested as a possible mechanism causing the impaired postural control [2].

When postural conditions change, the central nervous system (CNS) must identify and select the sensory inputs to provide good control. The CNS weight inputs from the vestibular, the visual and the proprioceptive system. So, to maintain a good equilibrium the task is to control efficiently the center of mass by muscle forces [2]. Thus, the postural control in lower back pain can be affected by factors such as reduction in somatosensory input when visual and vestibular inputs are intact. Also, postural control can be affected by other factors such as: age, external loads, localized muscle fatigue, neurological deficits, and musculoskeletal disorders (back pain) which reduce the quality of afferents [3]. There are a lot of studies which analyzing trunk control between different population [4], the effect of applied trunk forces [5], whole body vibration [6] or vibrotactile feedback devices [7], [8], or sensory manipulation on trunk control [9]. To increase trunk stability and deep muscles activity all these devices and strategies mentioned before are applied.

The most studies show unstable base of support, when center of mass changes during unstable sitting [10], [5], [11], [12] or standing [13]. There are several types of base have been use in unstable sitting: a central ball bearing [14], [15], a central ball and springs [5], [16], [12], [17], [10], and hemispherical bases [4], [18], [19]. The perturbation of postural control acts when trunk moves to different directions. The important features of unstable base of support, such as chairs with movable seat, are that it increases the range of the pelvis movement or forces the pelvis movement [20]. The more engaging the pelvis motion, the more engaging of the muscles supporting the spine [20].

\section{Testing procedures}

Our study is a pilot study, one subject (man) participated in our experiment. Age 40 years old, weight $80 \mathrm{~kg}$, height $-180 \mathrm{~cm}$.

The trunk muscles activity was measured during the balance testing by surface electromyograph (sEMG) (Noraxon MR3.6). Bipolar EMG activity was recorded using surface electrodes from the right side of: $\mathrm{m}$. transversus, $\mathrm{m}$. obliqus externus, $\mathrm{m}$. rectus abdominis, $\mathrm{m}$. erector spine (thoraco - lumbar part), m. multifidus. Before the surface electrodes placement on the skin was shaved, swabbed and rubbed with alcohol to reduce skin impedance. sEMG data were band-pass filtered (the frequency range 5-500 Hz) then rectified and smoothened. The amplitude of sEMG data was normalized using the mean dynamic activity method. For further calculations and results comparison between patients, maximum voluntary isometric contraction (MVIC) was performed for each muscle and the sEMG amplitude recorded at the same time.

Three-dimensional kinematic data were recorded by motion capture with 10 Oqus3+ (Qualisys AB, Gothenburg, Sweden) cameras Fig.1 and the Qualysis Track Manager software at a sampling frequency of $300 \mathrm{~Hz}$. The Helen-Hayes marker set was used for measuring full - body kinematics.

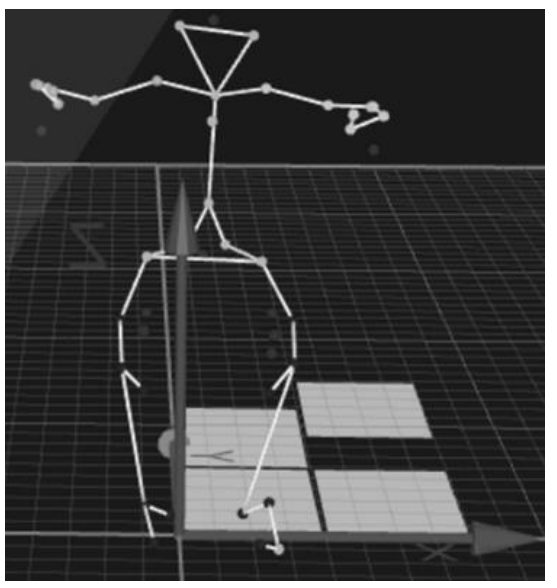

Fig. 1 Sitting human model with active markers 


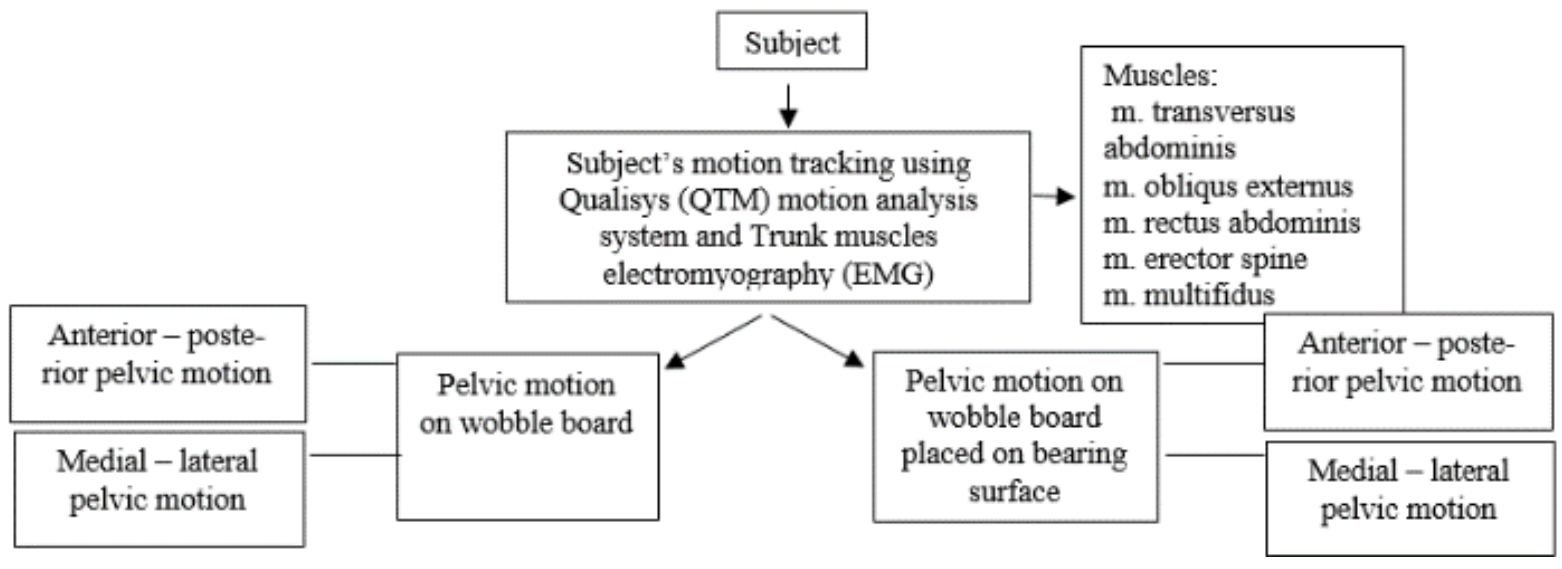

Fig. 2 Testing protocol

Experiment was done on testing protocol Fig. 2. Experimental task was done on two different sitting surface Fig. 3 with two pelvic motions on them. The first task was sit on wobble board Fig. 3 and do two pelvic motions: anterior - posterior pelvic motion and side to side pelvic motion. The second task was sit on wobble board which was placed on bearing surface Fig. 3, the pelvic motions were: anterior - posterior pelvic motion and side to side pelvic motion.

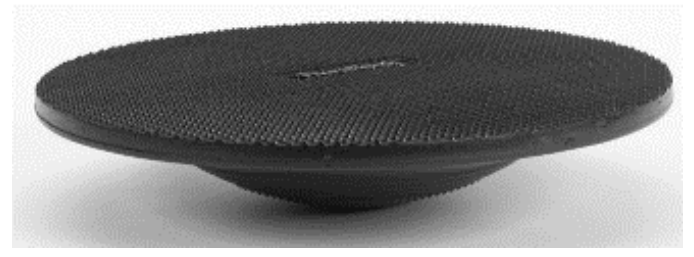

$\mathrm{a}$

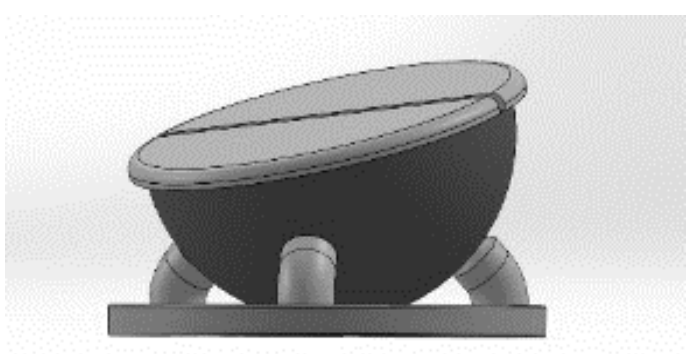

b

Fig. 3 a) a simple wobble board and b) wobble board placed on bearing surface

\section{Results}

\subsection{Trunk muscles activity on two different surfaces}

Person multifidus muscle activity acting anterior posterior pelvic motion on wobble board was $17,38 \%$, while sitting on wobble board on bearing surface was $23,07 \%$. Also, the multifidus muscle activity was higher $(23,07 \%)$ than erector spine muscles activity $(16,64 \%)$ on wobble board on bearing surface. Externus obliqus muscles activity $(20,83 \%)$ when sat on wobble board on bearing surface was higher than transversus $(13,77 \%)$ and rectus abdominis $(8,16 \%)$ muscles. Meanwhile, sitting on wobble board the abdominis muscles activity were lower than sitting on wobble board on bearing surface Fig. 4 .

During side to side pelvic motion, the multifidus muscles activity, were lower on wobble board on bearing surface $15,19 \%$ than on wobble board $21,28 \%$. Also, transversus muscle $(24,83 \%)$ and externus obliqus muscle $(29,82 \%)$ activity was higher on wobble board on bearing surface than on wobble board (transversus muscle $(18,41 \%)$ and externus obliqus muscle (19,68\%) Fig. 5.

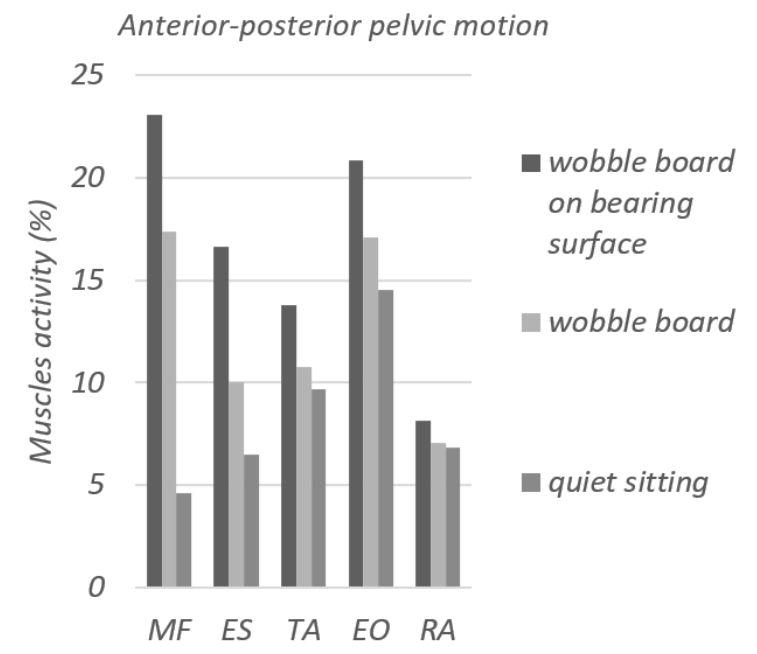

Fig. 4 Trunk muscles activity in anterior - posterior pelvic motion (MF - m. multifidus; ES - m. erector spine; TA - m. transversus abdominis; EO - m. obliqus externus; RA - m. rectus abdominis)

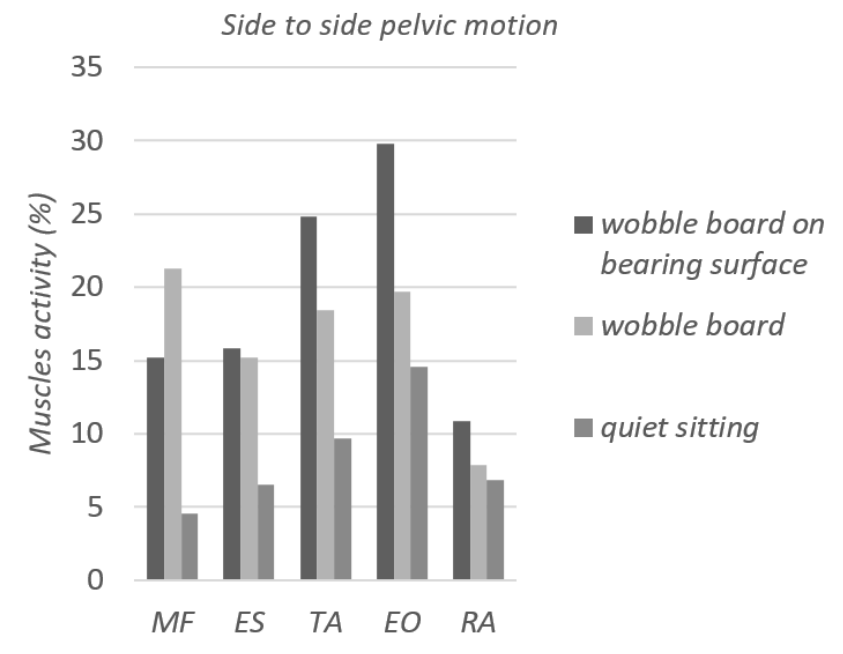

Fig. 5 Trunk muscles activity in side to side pelvic motion $(\mathrm{MF}-\mathrm{m}$. multifidus; $\mathrm{ES}-\mathrm{m}$. erector spine; TA - $\mathrm{m}$. transversus abdominis; EO - m. obliqus externus; $\mathrm{RA}-\mathrm{m}$. rectus abdominis) 


\subsection{Kinematic body segments analysis}

During anterior - posterior pelvic motion on wobble board on bearing surface, the degrees of pelvis related to sitting surface was lower $64,44^{\circ}$, than on wobble board $-70,81^{\circ}$ Fig.6. However, side to side pelvic motion on wobble board on bearing surface was higher $80,06^{\circ}$, than on wobble board $-86,20^{\circ}$ Fig. 7

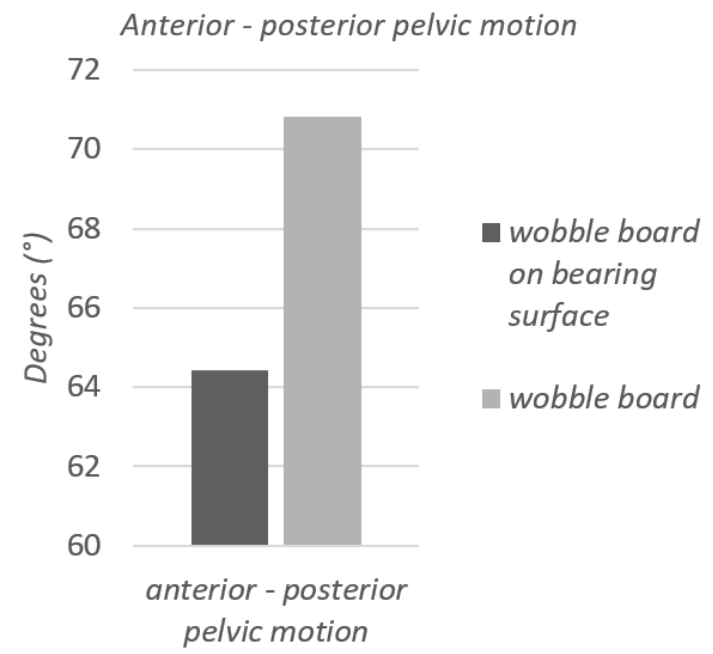

Fig. 6 Pelvis angle in anterior - posterior pelvic motion

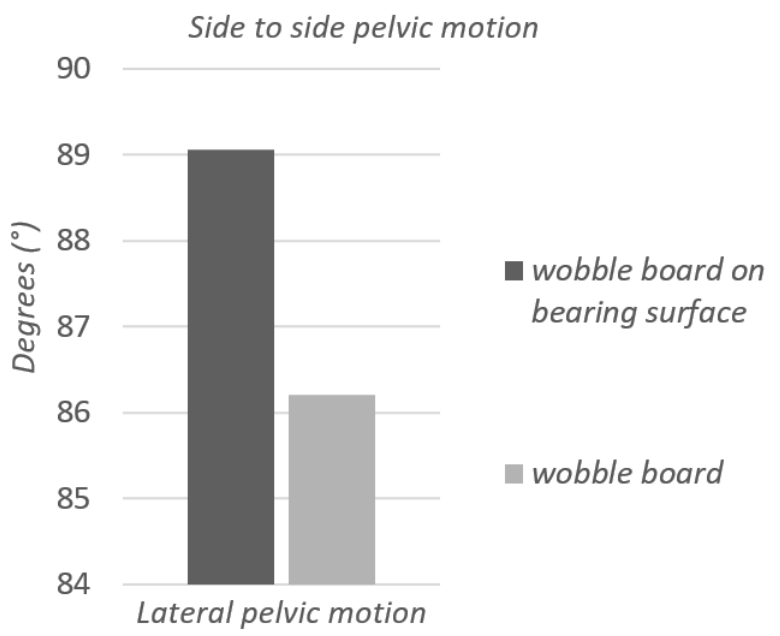

Fig. 7 Pelvis angle in side to side pelvic motion

\section{Discussion}

The purpose of this experiment was to compare person's trunk muscles activity and kinematic body segments movement when sitting on different platforms: simple wobble board and wobble board placed on bearing surface. The wobble board placed on bearing surface was selected due to find are the deep muscles more active than superficial muscles and how the angle of pelvis changes during movement on different surfaces. The main findings were that doing anterior - posterior pelvic motion on wobble board on bearing surface, all muscles had higher activity than doing the same motion on simple wobble board, but the anterior - posterior pelvis motion angle was lower on wobble boar on bearing surface, than on wobble board. The goal of the wobble board and person dynamic system is to stabilize the upper body. Mechanically, the wobble board is lighter, and of lower inertia compared to the upper body [16]. For this reason, we assume, that our body system is most effectively stabilized by regulating the motion of the wobble board placed on bearing surface due to higher activity of deep trunk muscles and lower angle of pelvic motion. This wobble board on bearing surface can be potential to train seated balance and pelvic motion in clinical populations [17], when neuromuscular control of pelvis and lumbar spine are affected and people with lower back pain cannot move pelvis isolated [18].

Talking about side to side pelvic motion muscles transversus abdominis and externus obliqus abdominis had higher activity on wobble board on bearing surface, than on simple wobble board. However, the higher angle, doing side to side pelvic motion, was on simple wobble board, than on wobble board on bearing surface.

This experiment used a hemispherical base which was placed on bearing surface of the sitting surface to induce unstable sitting and pelvic motion. Our findings of wobble board placed on bearing surface can be other type of unstable sitting idea.

\section{Conclusions}

1. Sitting on wobble board placed on bearing surface leaded to increment of the activity of deep muscles, which are responsible for spine stabilization, especially in anterior - posterior pelvic motion.

2. This bearing surface let to do smaller pelvis motion and gave higher muscles activity than on simple wobble board.

3. Wobble board placed on bearing surface could be adjusted to decrease superficial muscles activity and increase deep muscles activity, especially those people who have impaired neuromuscular control in lumbo - pelvic region. Nevertheless, there is still a need of further research, considering larger sample sizes and improvement of wobble board placed on bearing surface.

\section{References}

1. Andreopoulou, G.; Maaswinkel, E.; Cofré Lizama, L. E.; van Dieën, J. H. (2015). Effects of support surface stability on feedback control of trunk posture, Experimental Brain Research 233(4): 1079-1087. http://doi.org/10.1007/s00221-014-4185-5.

2. AU - Williams, A. D.; AU - Vette, A. H. 2019. A vibrotactile feedback device for seated balance assessment and training, JoVE, 143, e58611. http://doi.org/10.3791/58611.

3. Claeys, K.; Brumagne, S.; Dankaerts, W.; Kiers, H.; Janssens, L. 2011. Decreased variability in postural control strategies in young people with non-specific low back pain is associated with altered proprioceptive reweighting, European Journal of Applied Physiology 111(1): 115-123. http://doi.org/10.1007/s00421-010-1637-X.

4. Cruise, D. R.; Chagdes, J. R.; Liddy, J. J.; Rietdyk, S.; Haddad, J. M.; Zelaznik, H. N.; Raman, A. 2017. An active balance board system with real-time control of stiffness and time-delay to assess mechanisms of postural stability, Journal of Biomechanics 60: 48-56. http://doi.org/10.1016/j.jbiomech.2017.06.018.

5. Freddolini, M.; Strike, S.; Lee, R. 2014. Dynamic stability of the trunk during unstable sitting in people with low back pain, Spine, 39(10). Retrieved from 
https://journals.lww.com/spinejournal/Fulltext/2014/05 010/Dynamic_Stability_of_the_Trunk_During_Unstabl e.2.aspx doi: 10.1097/BRS.0000000000000296.

6. Freddolini, M.; Strike, S.; Lee, R. Y. W. 2014. Stiffness properties of the trunk in people with low back pain, Human Movement Science 36: 70-79. https://doi.org/10.1016/j.humov.2014.04.010.

7. Larivière, C.; Gagnon, D. H.; Mecheri, H. 2015. Trunk postural control in unstable sitting: effect of sex and low back pain status, Clinical Biomechanics 30(9): 933-939.

http://doi.org/10.1016/j.clinbiomech.2015.07.006.

8. Larivière, C.; Mecheri, H.; Shahvarpour, A.; Gagnon, D.; Shirazi-Adl, A. 2013. Criterion validity and between-day reliability of an inertial-sensor-based trunk postural stability test during unstable sitting, Journal of Electromyography and Kinesiology 23(4): 899-907. http://doi.org/10.1016/j.jelekin.2013.03.002.

9. Larivière, C.; Preuss, R.; Ludvig, D.; Henry, S. M. 2020. Is postural control during unstable sitting a proxy measure for determinants associated with lumbar stability, Journal of Biomechanics 102: 1-9. http://doi.org/10.1016/j.jbiomech.2019.109581.

10. Lee, H.; Granata, K. P.; Madigan, M. L. 2008. Effects of trunk exertion force and direction on postural control of the trunk during unstable sitting, Clinical Biomechanics 23(5): 505-509.

http://doi.org/10.1016/J.CLINBIOMECH.2008.01.003.

11. Marchal-Crespo, L.; Reinkensmeyer, D. J. 2009. Review of control strategies for robotic movement training after neurologic injury, Journal of NeuroEngineering and Rehabilitation 6(1). http://doi.org/10.10.1186/1743-0003-6-20.

12. Michnik, R.; Chrzan, M.; Wodarski, P.; Bieniek, A.; Nowakowska, K.; Pollak, A.; Mitas, A. 2018. Research on the stability of the users of chair with a spherical base, Advances in Intelligent Systems and Computing 623: 299-307.

http://doi.org/10.1007/978-3-319-70063-2_32.

13. Motealleh, A.; Kordi Yoosefinejad, A.; Ghoddosi, M.; Azhdari, N.; Pirouzi, S. 2019. Trunk postural control during unstable sitting differs between patients with patellofemoral pain syndrome and healthy people: A cross-sectional study, Knee 26(1): 26-32. http://doi.org/10.1016/j.knee.2018.10.002.

14. Oomen, N. M. C. W.; Reeves, N. P.; Priess, M. C.; van Dieën, J. H. 2015. Trunk muscle coactivation is tuned to changes in task dynamics to improve responsiveness in a seated balance task, Journal of Electromyography and Kinesiology 25(5): 765-772. http://doi.org/10.1016/j.jelekin.2015.07.001.

15. Roberts, B. W. R.; Vette, A. H. 2019. A kinematics recommendation for trunk stability and control assessments during unstable sitting, Medical Engineering and Physics 73: 73-76. http://doi.org/10.1016/j.medengphy.2019.08.004.

16. Seraj, M. S. M.; Sarrafzadeh, J.; Maroufi, N.; Takamjani, I. E.; Ahmadi, A.; Negahban, H. 2019. Comparison of postural balance between subgroups of nonspecific low-back pain patients based on O'Sullivan classification system and normal subjects during lifting, Archives of Bone and Joint Surgery 7(1): 52-60. http://doi.org/10.22038/abjs.2018.33737.1887.

17. Shahvarpour, A.; Shirazi-Adl, A.; Larivière, C. 2016. Active-passive biodynamics of the human trunk when seated on a wobble chair, Journal of Biomechanics, 49(6): 939-945.

https://doi.org/10.1016/j.jbiomech.2016.01.042.

18. Slota, G. P.; Granata, K. P.; Madigan, M. L. 2008. Effects of seated whole-body vibration on postural control of the trunk during unstable seated balance, Clinical Biomechanics 23(4): 381-386.

https://doi.org/10.1016/j.clinbiomech.2007.11.006.

19. van der Burg, J. C. E.; van Wegen, E. E. H.; Rietberg, M. B.; Kwakkel, G.; van Dieën, J. H. 2006. Postural control of the trunk during unstable sitting in Parkinson's disease, Parkinsonism and Related Disorders 12(8): 492-498. http://doi.org/10.1016/j.parkreldis.2006.06.007.

20. Williams, A. D.; Kumawat, A.; Agarwal, K.; Boser, Q. A.; Rouhani, H.; Vette, A. H. 2017. An instrumented wobble board for assessing and training dynamic sitting balance, IEEE International Conference on Systems, Man, and Cybernetics, SMC 2017, 2017Janua, 2249-2254. http://doi.org/10.1109/SMC.2017.8122955.

21. Willigenburg, N. W.; Kingma, I.; Van Dieën, J. H. 2013. Center of pressure trajectories, trunk kinematics and trunk muscle activation during unstable sitting in low back pain patients, Gait and Posture 38(4): 625-630. http://doi.org/10.1016/j.gaitpost.2013.02.010.

I. Aleknaite-Dambrauskiene, A. Domeika, V. Gudžiūnas, V. Zaveckas

\section{PILOT STUDY OF WOBBLE SEAT ON TRUNK MUSCLES ACTIVITY AND KINEMATICS}

S u m m a r y

This paper presents the pilot study of wobble seat on trunk muscles activity and kinematics. For this study the wobble board and wobble board on bearing surface were used to compare muscles activity and body segments kinematics on these different platforms. Two pelvic motions were performed: anterior - posterior and side to side. Results showed that sitting on wobble board placed on bearing surface leaded to increment of the activity of deep muscles with smaller pelvic movement in anterior - posterior pelvic motion.

Keywords: wobble board, wobble seat, sitting, bearings, muscles activity, kinematic analysis.

Received June 09, 2020

Accepted April 07, 2021 